\title{
The Proposal of QMS Implementation in Healthcare Office
}

\author{
Stefan Markulik and Anna Nagyova \\ Department of Safety and Quality Production, Faculty of Mechanical Engineering, Technical University of Kosice, Slovak
}

\begin{abstract}
Background: Application of Quality Management System (hereinafter QMS) according to ISO 9001 is a good precondition for increase of process efficiency inwards and customer confidence outwards. QMS application in the field of healthcare is less common than in other areas. However, the less common it is, the more doctors' offices and hospitals should attempt to increase the standard of management of activities and also their quality. Methods: Process of implementation can be divided into 3 separate phases, where each of one has its own characteristic. The main step is to implement requirements of ISO 9001 into the organization processes and help to meet customer /in this case patients/ demands. During this process is possible to use software tool, called MS Project which helps organization to get early perspective about system implementation. Results: After quality management implementation into the dentist office, the processes were clearly identified and all nonconformities according to ISO 9001 were noticed and reported. Conclusion: Processes in dentist office were clearly identified and the system provides the possibility to obtain customer feedback.
\end{abstract}

Key words: Quality, process approach, MS project, healthcare.

\section{Introduction}

Any change in management of any organization carries a great aversion from employees. Nevertheless, the aversion consequently lowers and new changes become a part of common life of an organization. Moreover, the fewer employees an organization has, the easier changes are applied into practice. It is not uncommon that there are some organizations which are so small that it is peculiar to address them as organizations, such as different doctors' offices in policlinics or dentists' offices. Particularly doctors' offices are specific because they do not have more than two employees, usually a treating physician and supplementary health assistance such as a nurse or a medical assistant. It is a commonly known fact that QMS implementation brings benefits for any organization, inwards or outwards. However, it is not clear how functioning of an organization differs if

Corresponding author: Anna Nagyova, Ing., PhD, lecturer, research fields: quality management system, project management. E-mail: anna.nagyova@tuke.sk.
QMS is applied in an environment which has a minimal number of employees [1].

\subsection{Patient as a Customer}

For understanding of quality management principle it is necessary to comprehend the term costumer orientation and the term customer. It is a common problem of an organization to identify its customers. To understand this point in the conditions of a dentist's office, it is important to answer the following question: Who is the customer of our dentist's office?

However, there is not just one answer to this question. Customer identification is not always as easy as it may seem because the view of a provider is limited (restricted), not seeing the broader relations which the dentist's office has with its customers. Customer can generally be defined as a person (natural or legal) to whom the dentist's office provides the results of its work. It is undeniable that dentist's office costumer is its patient - the person to whom professional medical care is provided (Fig. 1).

What happens if a patient comes into a dentist's office for a health check required by their employer? In 
this case the employer must also be considered as a costumer.

Regarding these questions, another group of (usually legal) persons has to be considered. The group consists of persons who are not direct costumers of dentists' offices but they are highly interested in the relation between the dentist's office and its patient. These are, for example, the Ministry of Health of the Slovak Republic, medical chamber, professional and trade unions etc. (Fig. 2).

What does it mean 'interested party' in the following context? This term represents all subjects which are interested in (accurate) functioning of a dentist's office.

\section{Methods - Quality Management System Implementation}

QMS implementation in a dentist's office does not differ significantly from implementation in large organizations. However, there could appear differences in time horizon as well as division of tasks within this kind.

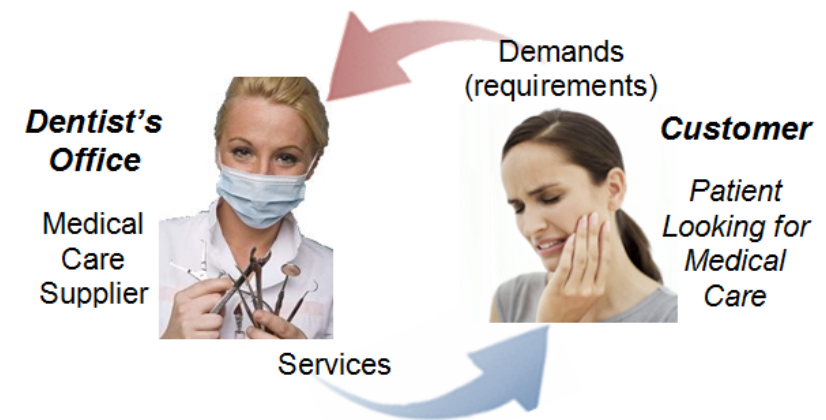

Fig. 1 Closer costumer-supply relationship.

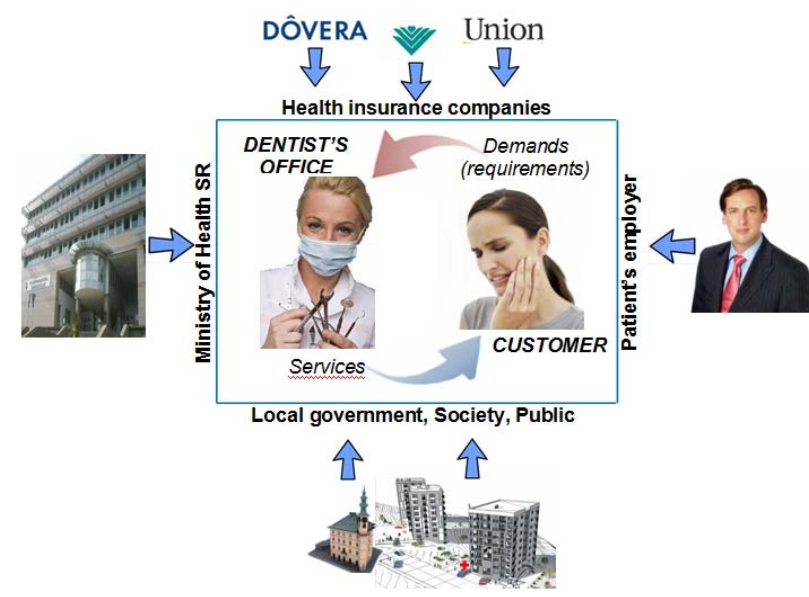

Fig. 2 Wider costumer-supply relationship.
The implementation is divided into three basic parts:

(1) Current status analysis;

(2) QMS documentation settings;

(3) Verification of QMS implementation;

Each step of implementation can be transformed into the software application, calls MS Project, which is designed to assist project managers in developing plans, assigning resources to tasks, tracking progress, managing budgets and analyzing workloads. Schedules can be resource leveled, and chains are visualized in a Gantt chart [4].

Planning of the implementation was performed with utilization of MS Project. Implementation time schedule, as an outcome of this program, was designed in the form of Gantt diagram (Fig. 3).

\subsection{Current Status Analysis}

Current status analysis examined control activities of the dentist's office hitherto, documentation management appropriateness and record keeping suitability. This phase identified the costumers and interested parties of the dentist's office as well as the necessity of vocational education of the dentist and supplementary health assistance in the field of quality management. Specific education of personnel is necessary for the right function of QMS. Base of this education is to appropriate manage record documentations.

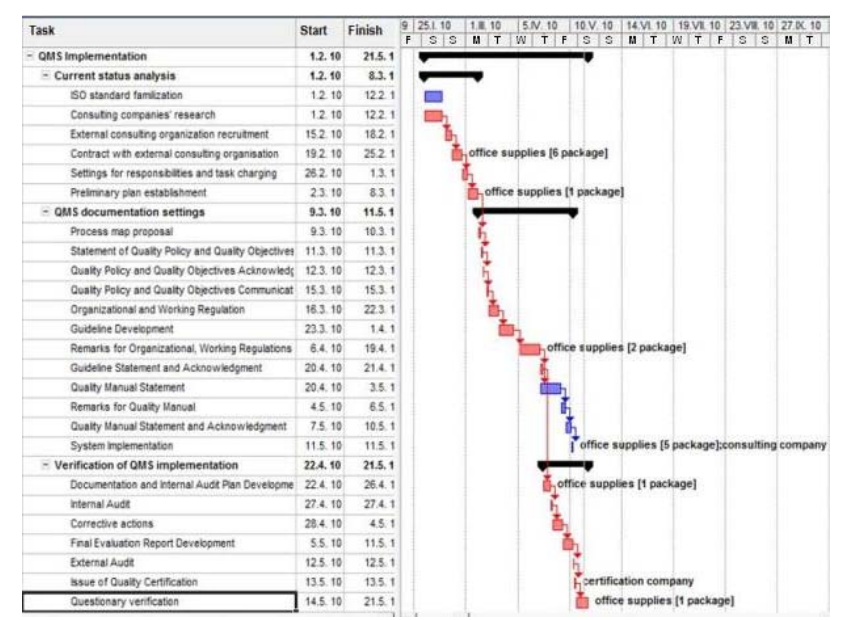

Fig. 3 Gantt diagram. 
The analysis also included a budget estimate which outlined probable QMS implementation costs. Each implementation phase was assigned resources which were consequently included in budget. The implementation process was coordinated with an external counseling organization what, of course, influenced overall costs - they were higher than if the implementation was self-performed (Fig. 4).

The implementation was planned for 78 days, what is a standard period of implementation process in organizations with such low number of employees. Total costs of mentioned process reached $€ 1.848$ (Fig. $5)$.

\subsection{QMS Documentation Setting}

QMS documentation setting began with identification of all processes of the dentist's office, their sorting into three groups (major, managerial, supportive) and their demonstration on following map (Fig. 6).

This map illustrates relation and interaction between particular processes. The map completion was followed by preparation of documents (directives) for

\begin{tabular}{c|c|r|}
\hline & Task & Total cost \\
\hline 1 & - QMS Implementation & 1848,00 EUR \\
\hline 2 & + Current status analysis & 21,00 EUR \\
\hline 9 & + QMS documentation settings & 1021,00 EUR \\
\hline 22 & + Verification of QMS implementation & 806,00 EUR \\
\hline
\end{tabular}

Fig. 4 Financial costs by phase.

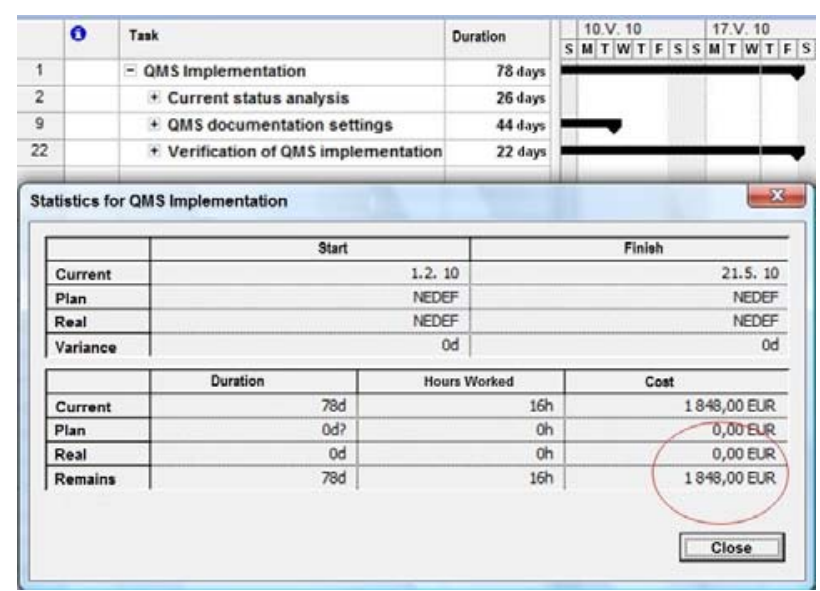

Fig. 5 Time schedule and financial plan of the implementation. accomplished process standardization. Mentioned documentation included two sets of rules (work and organizational), which, e.g., provide description of particular responsibilities and competences attached to concrete positions (of a dentist and a nurse).

EN ISO 9001 standard requires documented procedures for:

- Control of documents,

- Control of records,

- Control of nonconforming product,

- Internal audit,

- Corrective action,

- Preventive action [1].

The form for control of documents and control of records were coupled into one regulation because both are closely linked. Give strict rules for all documents. Furthermore, control of nonconforming product is covered in a separate directive while internal audit management is described in a quality manual. Because the dentist's office does not have its own auditor it has to outsource this process. By contrast, internal resource planning was performed by the dentist himself. The dentist's office cannot get rid of responsibility only because some activities are performed by an external person (auditor). Similarly to the control of documents and records, corrective and preventive actions were also coupled into one directive [2].

Resource management process, which is depicted in the process map, is outlined in a separate directive which describes the competence of employees (the dentist and the nurse), training plans and evaluation of their efficiency. It moreover determines the infrastructure which is necessary for an accurate nursing performance. The term infrastructure in the conditions of the dentist's office includes care for its interior, equipment and software used for record keeping. Resource management also involves description of work environment conditions (temperature, lighting, cleanness, sterility) [3].

The key documentation of implemented QMS was dental treatment documentation. It presents procedures 
of professional advice (which is depicted as a separate process in the process map). This directive standardizes the dental treatment procedure, i.e., it specifies its steps starting with registration of a patient, consultancy about following treatment and determination of its procedure if it is necessary.

The treatment cannot be performed if a patient does not state a clear agreement with the procedure. Dentist's office should highly focus on the social aspect of examination. Since the visit of a dentist is generally connected with unpleasant feelings, dentist's perception of a patient is very important, especially in the case of patient's discomfort. In the end of the examination the patient is advised on dental and mouth hygiene.

Purchasing and Contractual Relationship directives describe procedures for performance of these processes with regard to quality of provided services. The Purchasing process includes purchasing of different material ranging from office supplies to tools for dental treatment. The Contracting relation directive outlines the providing of incorporated procedure within these relations, their evaluation or facture and recovery of claims which could arise within the functioning of the dentist's office.

\section{Results - Verification of QMS Implementation}

The most important benefit of QMS application in the dentist's office is the change of customer's perception. The change was positive because customer orientation, the key aspect of QMS, is visible immediately after entering the dentist's office. The dentist is focused on communication with customer from beginning till the end of the visit. The customer (patient) can declare their (dis)satisfaction in a questionnaire which is easily accessible and visibly marked. Due to low interest of patients in expressing their opinion, customer satisfaction is evaluated once in six months. During the evaluation the dentist found out that patients are not used to expressing their satisfaction in a formal way (the questionnaire). Many patients understand their satisfaction expression in paying the surcharge for treatment in the dentist's office. Within satisfaction evaluation (once in six months) the dentist draws three patients who are awarded with beforehand selected benefits. The benefits represent discounts of different value on services offered by the dentist's office. Their character changes every six months in order to keep them interesting for patients. Generally, according to the dentist's opinion, activities in the dentist's office are better arranged. Processes are understood as mutually dependant actions which outcome is satisfied customer (patient).

\section{Conclusion}

QMS implementation is a difficult process from time and organizational point of view because it requires knowledge on ISO 9001 standard and understanding its consequences for the dentist's office.

Computer support (MS Project) used within QMS implementation is really helpful for dentist's office owners. The main advantage of this kind of software application is the fact that it displays outline of drawn resources and completed actions in contrast with the plan. During project realization it also enables to control the "substantial" performance, i.e., which of planned actions have been completed or phase of their completion - created value and real cost incurred so far within their implementation.

\section{Acknowledgements}

The research which lead to these results, achieved financial capital from Seventh Frame program iNTegRisk based on Grand agreement Nr. CP-IP 213345-2 and along with fund up Slovak Research and Development Agency accordung to Agreement $\mathrm{Nr}$. DO7RP-0019-08. 


\section{References}

[1] Š. Markulik and A. Nagyová, Quality Management System, Košice, 2009.

[2] S. Markulik and A. Nagyová, Quality as a main aspect of Healthcare, in: YBERC 2010 Young Biomedical Engineerings and Researchers Conference, July 1-3, 2010.

[3] A. Sütőová and M. Šolc, Utilization of statistical methods within the European performance satisfaction index (EPSI) rating methodology, in: Kvalita Inovácia Prosperita. Roč. 13, č. 1 (2009), s. 77-82.

[4] A. Nagyová and H. Pačaiová, Quality evaluation methodology for research projects, in: DAAAM International Scientic Book 2010, 21th International Conference, 2010, pp. 219-226.

[5] B. Hayes, Measuring Customer Satisfaction, United States of America: ASQ, 2008. 